\title{
A novel function of CYP21A2 in regulating cell migration and invasion via Wnt signaling
}

\author{
Yajie Guo ${ }^{1}$, Aliya Rehati ${ }^{2}$, Zhijing Wu ${ }^{1}$, Wenyuan Zhang ${ }^{1}$, Peng Zhuang ${ }^{1}$ and Fangping $\mathrm{He}^{1}$ \\ ${ }^{1}$ The Eighth Affiliated Hospital, Sun Yat-Sen University, Shenzhen, Guangdong province, China \\ ${ }^{2}$ Department of Hepatology, The First Affiliated Hospital of Xinjiang Medical University, Urumchi, Xinjiang province, China
}

\begin{abstract}
CYP21A2, which is responsible for 21-hydroxylase activity, is prominent to the development of congenital adrenal hyperplasia (CAH). The aim of our current study is to investigate the role of CYP21A2 in the tumor processes. Here, we used HepG2 cell lines and generated CYP21A2 overexpressing vector and siRNA to investigate the effect of CYP21A2 on the tumor development processes, particularly cell migration and invasion; genes expression related to these processes were further examined. Results showed that CYP21A2 over-expressed or silenced had no effects on cell viability as well as the process of cell apoptosis. Further study suggested that CYP21A2 silenced significantly decreased the G0/G1 phase and increased the S phase of the cell cycle. However, no differences were observed when CYP21A2 was overexpressed. Moreover, we found that cell migration and invasion significantly improved with CYP21A2 overexpressed and impaired with silenced CYP21A2. Finally, we examined the expression of genes related to tumor processes and found that the Wnt signaling genes were changed. Taken together, our results demonstrated a novel function of CYP21A2 in the regulation of tumor processes, particularly cell migration and invasion, which this may be mediated by the Wnt signaling pathway.
\end{abstract}

Key words: CYP21A2 - Cell migration and invasion - Wnt signaling

Abbreviations: $\mathrm{CAH}$, congenital adrenal hyperplasia; Ccnd2, cyclin D2; Cox17, cytochrome c oxidase assembly homolog 17; Ctnnb1, catenin beta 1; Cyp19a1, cytochrome P450 family 19 subfamily A member 1; CYP21A2, cytochrome P450 21-hydroxylase; DKK1, Dickkopf WNT signaling pathway inhibitor 1; Egfr, epidermal growth factor receptor; Tcf7, transcription factor 7; Gpc3, glypican 3; Hifla, hypoxia inducible factor 1 subunit alpha; Hsp70, heat shock protein family A member 5; Hyou1, hypoxia up-regulated 1; Myc, MYC proto-oncogene; Ndufa2/Ndufa3, NADH: ubiquinone oxidoreductase subunit A2/A3; Nos3, nitric oxide synthase 3; Sec63, Sec63 homolog, protein translocation regulator.

\section{Introduction}

Cancer is responsible for one of the major causes to death which is believed to be one end result of a process of somatic evolution (Hahn and Weinberg 2002; Calcinotto et al. 2019;

Electronic supplementary material. The online version of this article (doi: 10.4149/gpb_2020004) contains supplementary material, which is available to authorized users.

Correspondence to: Fangping He, 3025 Shennan middle Road, Shenzhen, Guangdong Province, China 518033

E-mail: hefp@sina.com
Siegel et al. 2019; Srivastava et al. 2019). It is related to genetic mutations or environmental factors which endow a single clonal lineage cells with adaptive and proliferative advantages (Martincorena et al. 2018; Puisieux et al. 2018). Several processes promoted tumor development including cell viability, proliferation, apoptosis, migration, invasion and also angiopoiesis (Strilic and Offermanns 2017; Puisieux et al. 2018). Numerous studies have demonstrated kinds of signaling pathways and regulators that were required for the formation and development of tumor, including Wnt signaling (Ghosh et al. 2019; VanderVorst et al. 2019), ERK signaling (Lito et al. 2013), Notch signaling (Meurette and 
Mehlen 2018), SUMOylation (Eifler and Vertegaal 2015) and some others (Schmitt and Chang 2016; Rojo de la Vega et al. 2018; Neagu et al. 2019). Although many regulatory mechanisms regarding these processes have been identified up to date, detailed and novel mechanisms require to be explored.

CYP21A2 (cytochrome P450 21-hydroxylase) is a member of the cytochrome P450 superfamily (Canturk et al. 2011), which includes highly versatile heme containing enzymes catalyzing a variety of oxidation reactions (Bernhardt 2006; Lamb and Waterman 2013; Girvan and Munro 2016). As a vital regulator for 21-hydroxylase activity (Carmina et al. 2017), CYP21A2 plays an important role in the development of congenital adrenal hyperplasia (CAH) (Canturk et al. 2011; Haider et al. 2013; New et al. 2013; Carmina et al. 2017). About $95 \%$ of CAH cases are caused by deficiency of 21-hydroxylase activity (Canturk et al. 2011). CYP21A2 is a protein located in the endoplasmic reticulum and crucial in steroid hormone biosynthesis that provides the precursors of aldosterone, glucocorticoid, cortisol and mineralocorticoid (Ryan and Engel 1957; Kominami et al. 1980; Mizrachi et al. 2011; Brixius-Anderko et al. 2015). Previous studies showed that CYP21A2 was involved in the regulation of physiological processes, including congenital adrenal hyperplasia (Carmina et al. 2017), polycystic ovarian syndrome, infertility (Bry-Gauillard et al. 2014) and hypertension (Lu et al. 2015). Recently, however, studies implied a possible role of CYP21A2 in the regulation of tumor metabolism (Charni et al. 2016). From microarray analysis of liver cells, it was found that CYP21A2 may be a novel target gene of p53, an important tumor suppressor that functions to prevent tumor development (Vogelstein et al. 2000). The above study suggested that CYP21A2 may play an important role in cancer development. The aim of our current study is to investigate this possibility and implore the underlying mechanism.

\section{Materials and Methods}

\section{Cell lines}

The human hepatoma cell line HepG2 was obtained from KeyGEN BioTECH (Nanjin, Jiangsu Province). Cells were maintained in Dulbecco's modified Eagle's medium (DMEM) (KeyGEN BioTECH, Nanjin, China) with 10\% fetal bovine serum (FBS) (Gibco, Invitrogen Corp.), 1\% penicillin and streptomycin (Invitrogen Corp.). All cell lines were cultured in a humidified incubator $\left(5 \% \mathrm{CO}_{2} / 95 \%\right.$ air atmosphere at $\left.37^{\circ} \mathrm{C}\right)$.

\section{Plasmids construction and cell treatments}

The DNA fragment encoding CYP21A2 was amplified from human HepG2 cells cDNA. The DNA fragment was then cloned to the control vector pIRES2 EGFR to form the CYP21A2 expressing plasmid. Double-stranded siRNA targeting human CYP21A2 was purchased from GenePharma. The siRNA sequence specific for human CYP21A2 is following: 5'-CCCUGCUCUGGAAAGCCCACAAGAA-3'. HepG2 cells were transfected with CYP21A2 expressing plasmid by Lipofectamine 3000 (Life technologies) or CYP21A2 siRNA by Lipofectamine RNAiMAX Transfection Reagent (Life technologies), respectively.

\section{CCK-8 assay}

Cell cytotoxicity was evaluated by CCK- 8 assay according to the manufacturer's instructions (TransGen Biotech, Beijing, China). HepG2 cells were seeded in a 96-well plate at a density of $5 \times 10^{3}$ cells per well. After incubation for $24 \mathrm{~h}$, cells were transfected with CYP21A2 expressing plasmid or siRNA or the controls, respectively. After another 24-h incubation, the medium removed and 10\% CCK- 8 assay solution added to each well. Cells were further incubated for $2 \mathrm{~h}$ and the absorbance was measured using a Microplate Reader at the wavelength of $450 \mathrm{~nm}$.

\section{Cell cycle detection}

HepG2 cells were seeded in $25 \mathrm{~cm}^{2}$ culture bottles at a density of $1 \times 10^{5}$ cells $/ \mathrm{ml}$. After incubation for $24 \mathrm{~h}$, cells were transfected with CYP21A2 expressing plasmid or siRNA or the controls. After another 24-h incubation, the medium removed, then cells were washed twice with PBS and digested using trypsin (Gibco, Invitrogen Corp.). The harvested cells were fixed overnight in $4^{\circ} \mathrm{C}$ with cold PBS and $75 \%$ ethyl alcohol. And then washed twice with cold PBS, added PI/RNase staining buffer and filtrated using 200 mesh nylon screen. After $30 \mathrm{~min}$ incubated in dark, the red fluorescence at the excitation wavelength of $488 \mathrm{~nm}$ were measured using Flow cytometry and analyzed the DNA content using software.

\section{Cell apoptosis detection}

Annexin V-FITC/PI apoptosis detection was made according to the manufacturer's instructions. HepG2 cells were seeded in $25 \mathrm{~cm}^{2}$ culture bottles at a density of $1 \times 10^{5} \mathrm{cells} / \mathrm{ml}$. After incubation for $24 \mathrm{~h}$, cells were transfected with CYP21A2 expressing plasmid or siRNA or the controls. $24 \mathrm{~h}$ later, the medium removed, cells were washed twice with PBS and then digested using trypsin (Gibco, Invitrogen Corp.). Next, cells were collected and washed twice with cold PBS and suspended in Annexin V binding buffer, filtrated using 200 mesh nylon screen and incubated with FITC labeled Annexin V for 15 min at $4^{\circ} \mathrm{C}$ in dark. Then, propidium iodide (PI) was added to incubate $5 \mathrm{~min}$ 
at $4^{\circ} \mathrm{C}$ in dark. The samples were analyzed by Flow cytometry (30 $\mathrm{min})$.

\section{Cell migration and invasion detection}

Cell migration and invasion analysis were measured using a transwell chamber containing $8 \mu \mathrm{m}$ pores (Corning, USA). HepG2 cells were transfected with CYP21A2 expressing plasmid or siRNA or the controls for $24 \mathrm{~h}$. Then cells were collected in serum free medium in a density of $5 \times 10^{5}$ cells $/ \mathrm{ml}$. The cells were plated into the upper chamber, and the lower chamber putted with medium supplement with 10\% FBS. After 72-h incubation, cells on the upper chamber were washed with PBS twice and stained with Giemsa solution (Nanjing Jiancheng Bioengineering Institute, Nanjing, China) and then counted in five fields randomly.

\section{$R N A$ isolation and relative quantitative RT-PCR}

mRNA levels were examined by RT-PCR using SYBR green fluorescent nucleic acid stain with the sequences of primers described in Table S1 (see Supplementary materials).

\section{Statistics}

All data are expressed as mean \pm SEM. Significant differences were assessed either by two-tailed Student $t$-test or one-way ANOVA followed by the Student-Newman-Keuls (SNK) test as indicated. A value $p<0.05$ was considered statistically significant.

\section{Results}

\section{CYP21A2 has no effect on cell viability}

To investigate the role of CYP21A2 in cell viability, we transfected HepG2 cells with plasmid expressing CYP21A2 (+ CYP21A2) or control vector (- CYP21A2) and then conducted the CCK- 8 assay. As expected, the expression of CYP21A2 increased dramatically with CYP21A2 overexpressing plasmid (Fig. 1A), but not affected cell viability (Fig. 1B). On the other hand, CYP21A2 silenced also had no effect on cell viability (Fig. 1D), although the expression of CYP21A2 was significantly decreased (Fig. 1C).

\section{CYP21A2 has no effect on cell apoptosis}

To further investigate the role of CYP21A2 in cell apoptosis, we transfected cells with CYP21A2 overexpressing vector or siRNA or the controls respectively, and then used flow cytometry to analyze the rate of cell apoptosis (Fig. 2A and C).

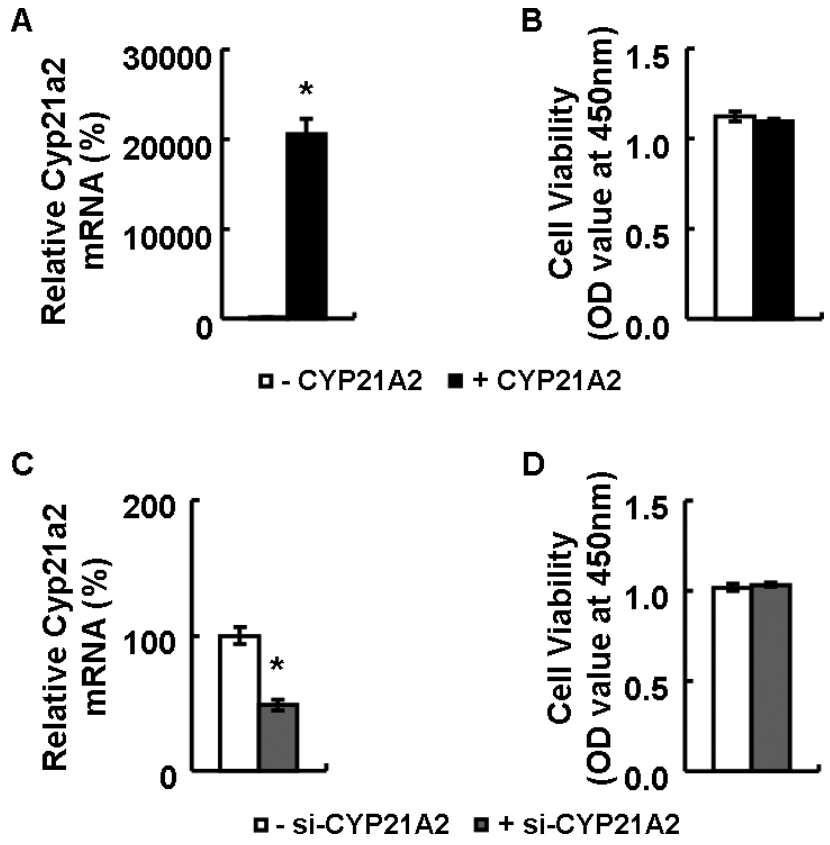

Figure 1. Effects of CYP21A2 on cell viability. Cells were transfected with CYP21A2 overexpressing plasmid (+ CYP21A2), control vector (- CYP21A2), CYP21A2 siRNA (+ si-CYP21A2), control siRNA (- si-CYP21A2) for $24 \mathrm{~h}$, respectively. A, C. CYP21A2 mRNA levels. B, D. CCK-8 assay. Data were obtained with at least three independent in vitro experiments $(n=10$ per group) and presented as means \pm SEM. Statistical significance was calculated using two-tailed Student's $t$-test for the effects of CYP21A2 versus control vector, si-CYP21A2 versus control siRNA. ${ }^{*} p<0.05$.

We examined the early apoptosis rate, the later apoptosis rate and the total apoptosis rate but found no differences no matter increased or decreased the expression of CYP21A2 (Fig. 2B and D).

\section{Knockdown of CYP21A2 changes cell cycle distribution}

Above results showed that CYP21A2 had no effects on cell viability and apoptosis. To further investigate its function on cell cycle, we overexpressed CYP21A2 and analyzed cell cycle distribution using flow cytometry. The results showed that there were no differences (Fig. 3A and B). Furthermore, we detected the protein levels of cell cycle G1-associated proteins like cyclin D and cyclin E. We found that the two protein expressions were not affected by CYP21A2 overexpressed (Fig. 3C). To confirm the role of CYP21A2 in cell cycle, CYP21A2 expression was then silenced. Surprisingly, we found that the cell cycle changed (Fig. 3D), the G0/G1 phase decreased and the S phase increased while the G2/M phase was not changed (Fig. 3E). Moreover, 
A
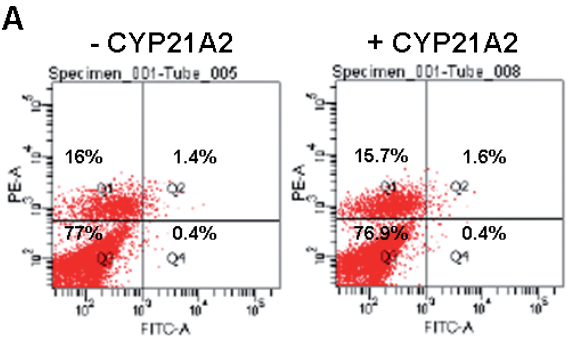

C

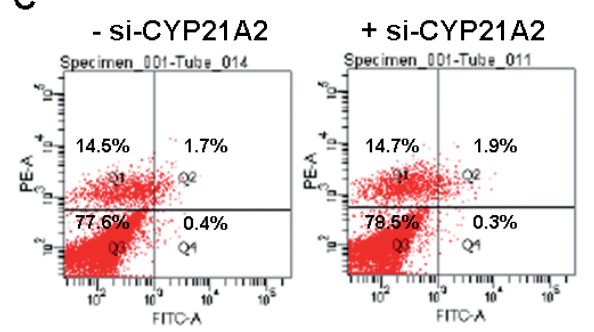

B

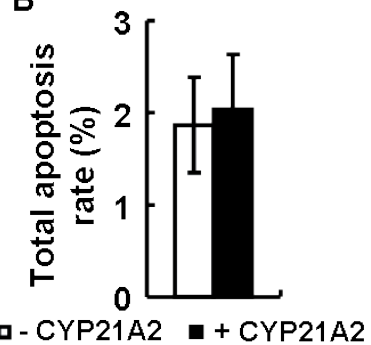

D

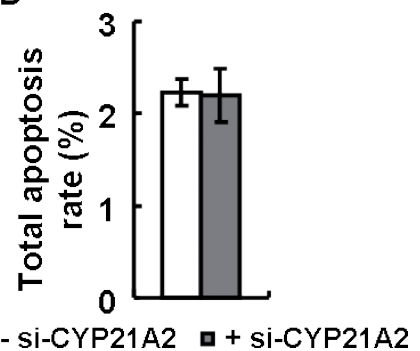

Figure 2. Effects of CYP21A2 on cell apoptosis. Cells were transfected with CYP21A2 overexpressing plasmid (+ CYP21A2), control vector (- CYP21A2), CYP21A2 siRNA (+ si-CYP21A2), control siRNA (- si-CYP21A2) for $24 \mathrm{~h}$, respectively. A, C. Results of flow cytometry scatter plot. B, D. Total apoptosis rate. Data were obtained with at least two independent in vitro experiments ( $n=6$ per group) and presented as means \pm SEM. Statistical significance was calculated using two-tailed Student's $t$-test for the effects of CYP21A2 versus control vector, si-CYP21A2 versus control siRNA. cyclin D expression was not changed but cyclin E expression was increased when CYP21A2 was downregulated (Fig. 3F). These results suggested that CYP21A2 may have a regulation role on cell proliferation. To confirm this, we did the cell proliferation assay but found no changes (Fig. S1, Supplementary materials).
A

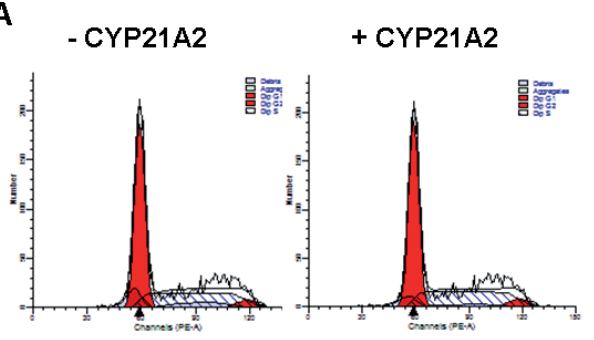

D

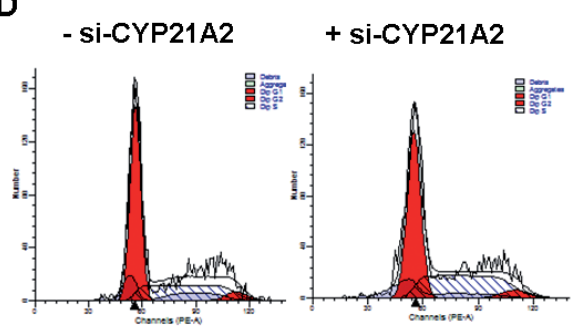

B

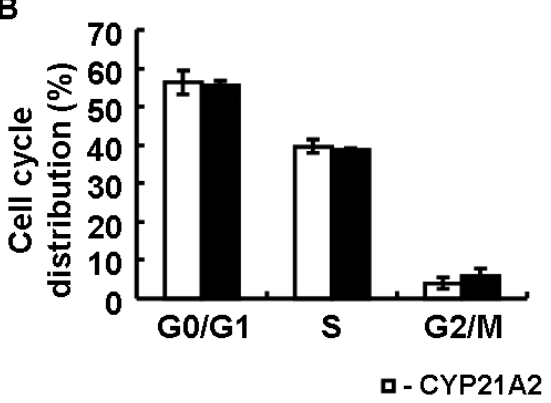

E

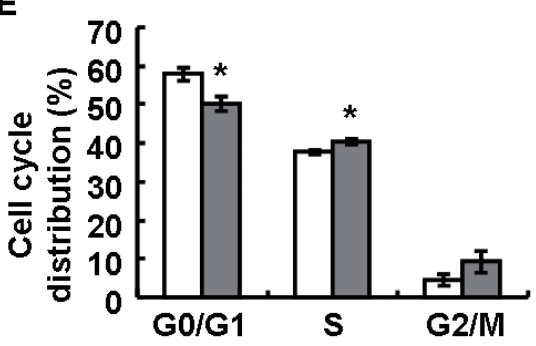

C

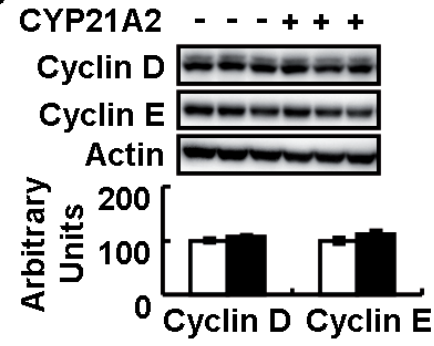

$F$

si-CYP21A2 - - + ++

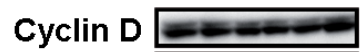

Cyclin E

Actin

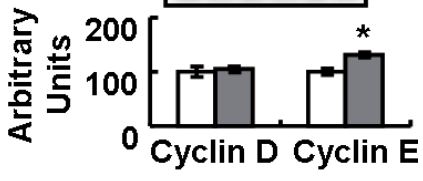

口- Si-CYP21A2 a+ si-CYP21A2

Figure 3. Effects of CYP21A2 on cell cycle. Cells were transfected with CYP21A2 overexpressing plasmid (+ CYP21A2), control vector (- CYP21A2) for 24 h, respectively. A, D. Flow cytometry results. B, E. Cell cycle distribution. C, F. Cyclin D and Cyclin E protein (top, Western blot; bottom, quantitative measurements of Cyclin D and Cyclin E protein relative to actin). Cells were transfected with CYP21A2 siRNA (+ si-CYP21A2), control siRNA (- si-CYP21A2) for $24 \mathrm{~h}$, respectively. Data were obtained with at least two independent in vitro experiments ( $n=6$ per group) and presented as means \pm SEM. Statistical significance was calculated using two-tailed Student's $t$-test for the effects of CYP21A2 versus control vector, si-CYP21A2 versus control siRNA. ${ }^{\star} p<0.05$. 


\section{CYP21A2 regulates cell migration and invasion}

Previous study had shown that CYP21A2 may regulate cell cycle, which plays a vital role in the process of tumor and cancer (Zhu and Thompson 2019). What's more, cell migration and invasion are also very important to tumor development (Cooper and Giancotti 2019). Next, we examined whether CYP21A2 regulate cell migration and invasion. We observed that CYP21A2 overexpressing markedly improved the migration ability and invasion ability of HepG2 cell following by the transwell assay (Fig. 4A and B). On the other hand, cell migration and invasion ability were significantly impaired when silenced CYP21A2 expression (Fig. 4C and D).

\section{CYP21A2 regulates genes expression related to tumor process}

Tumor development is related to several processes including Wnt signaling pathway, angiogenesis, cell proliferation and migration, protein modification in endoplasmic reticulum, oxidative phosphorylation, etc. (Clarke et al. 2012; De Palma et al. 2017; Lee et al. 2017; Ghosh et al.
2019; Zhu and Thompson 2019). We therefore examined the effects of CYP21A2 on genes expressions which were related to these processes in HepG2 cells overexpressing or silenced CYP21A2. The Wnt signaling genes like Ctnnb1 (catenin beta 1), Myc (MYC proto-oncogene) and DKK1 (Dickkopf WNT signaling pathway inhibitor 1) increased with CYP21A2 overexpressed and decreased with silenced CYP21A2 (Fig. 5A and D). The expression of other genes related to this pathway like Wnt6 (Wnt family member 6) and Ccnd2 (cyclin D2) were not changed with CYP21A2 overexpressed (Fig. 5A) or decreased with CYP21A2 silenced (Fig. 5D). What's more, the expression of Tcf7 (transcription factor 7) and Gpc3 (glypican 3) had no differences in two conditions (Fig. 5A and D). Genes related to angiogenesis and protein modification processes including Hifla (hypoxia inducible factor 1 subunit alpha), Vegfa (vascular endothelial growth factor A), Egfr (epidermal growth factor receptor), Nos3 (nitric oxide synthase 3), Sec63 (Sec63 homolog, protein translocation regulator), Hyou1 (hypoxia up-regulated 1) and Hspa5 (heat shock protein family A (Hsp70) member 5) were not changed or decreased (Fig. 5B and E). On the other hand, oxidative
A
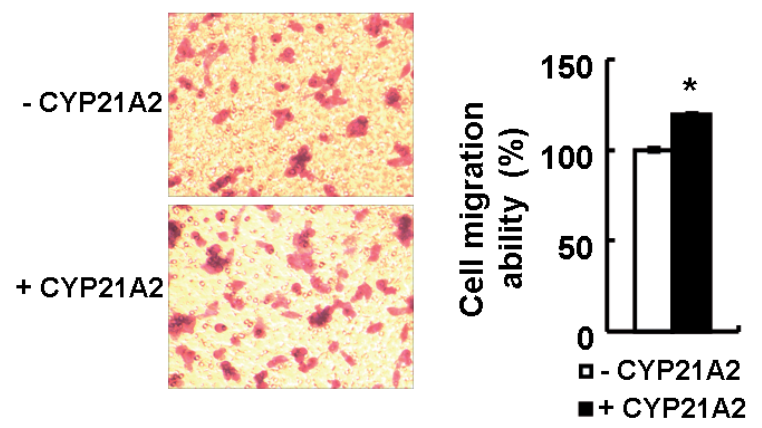

C

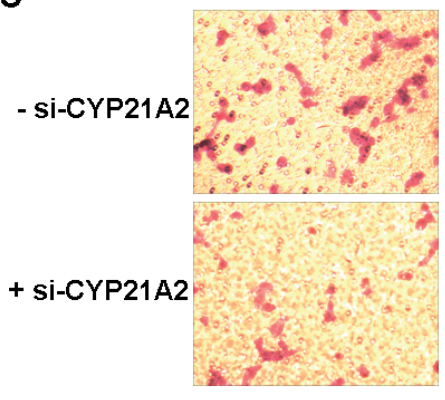

B
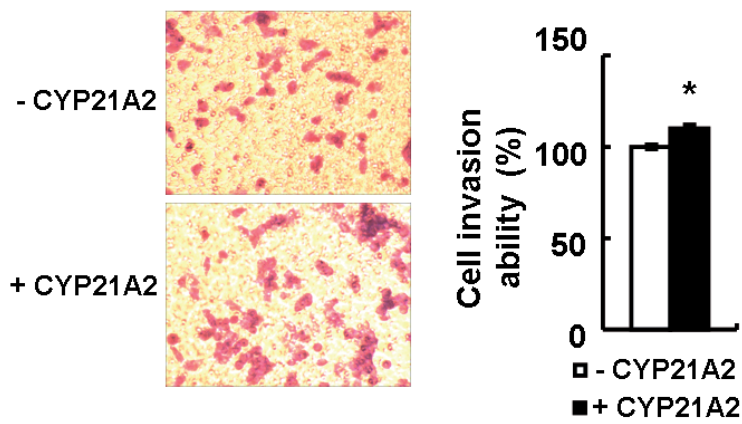

D

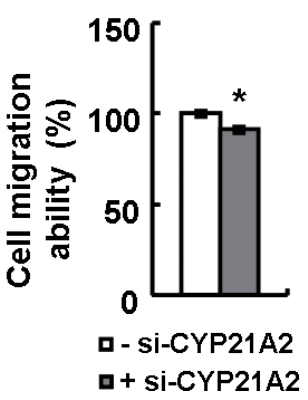

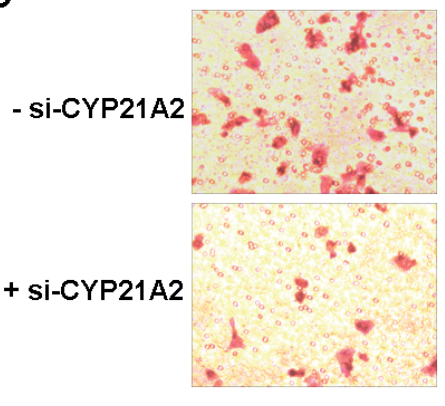

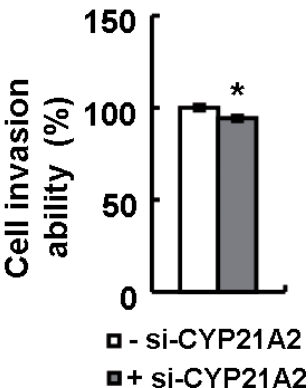

Figure 4. Effects of CYP21A2 on cell migration and invasion. Cells were transfected with CYP21A2 overexpressing plasmid (+ CYP21A2), control vector (- CYP21A2) for $24 \mathrm{~h}$, respectively. A, C. Transwell of cell migration assay. B, D. Transwell of cell invasion assay (left, Giemsa staining; right, relative level). Cells were transfected with CYP21A2 siRNA (+ si-CYP21A2), control siRNA (- si-CYP21A2) for $24 \mathrm{~h}$, respectively. Data were obtained with at least three independent in vitro experiments ( $n=60$ per group) and presented as means \pm SEMs. Statistical significance was calculated using two-tailed Student's $t$-test for the effects of CYP21A2 versus control vector, si-CYP21A2 versus control siRNA. ${ }^{\star} p<0.05$. 
phosphorylation in mitochondria is very important to tumor process which provides energy for cell proliferation, migration and invasion (Fulda et al. 2010; Green et al. 2014). We also detected the related genes expressions and found that Ndufa2 (NADH: ubiquinone oxidoreductase subunit A2), Ndufa3 (NADH: ubiquinone oxidoreductase subunit A3) and Cox17 (cytochrome c oxidase assembly homolog 17) showed no differences with CYP21A2 overexpressed (Fig. 5C) and decreased with CYP21A2 silenced (Fig. 5F). However, the expression of Cyp19a1 (cytochrome P450 family 19 subfamily A member 1), which is responsible for cell oxidation reaction, was decreased when CYP21A2 overexpressed (Fig. 5C) and increased when CYP21A2 silenced (Fig. 5F). We also examined the protein levels of Wnt signaling genes like Ctnnb1, Myc and DKK1 and found that these were increased under CYP21A2 overexpressed condition (Fig. S2A) but not changed under CYP21A2 silenced condition (Fig. S2B).

\section{Discussion}

Most of CYP21A2 functions known are related to congenital adrenal hyperplasia, one of the most frequent autosome recessive disorders (Carmina et al. 2017; El-Maouche et al. 2017), but the functions of CYP21A2 in other areas
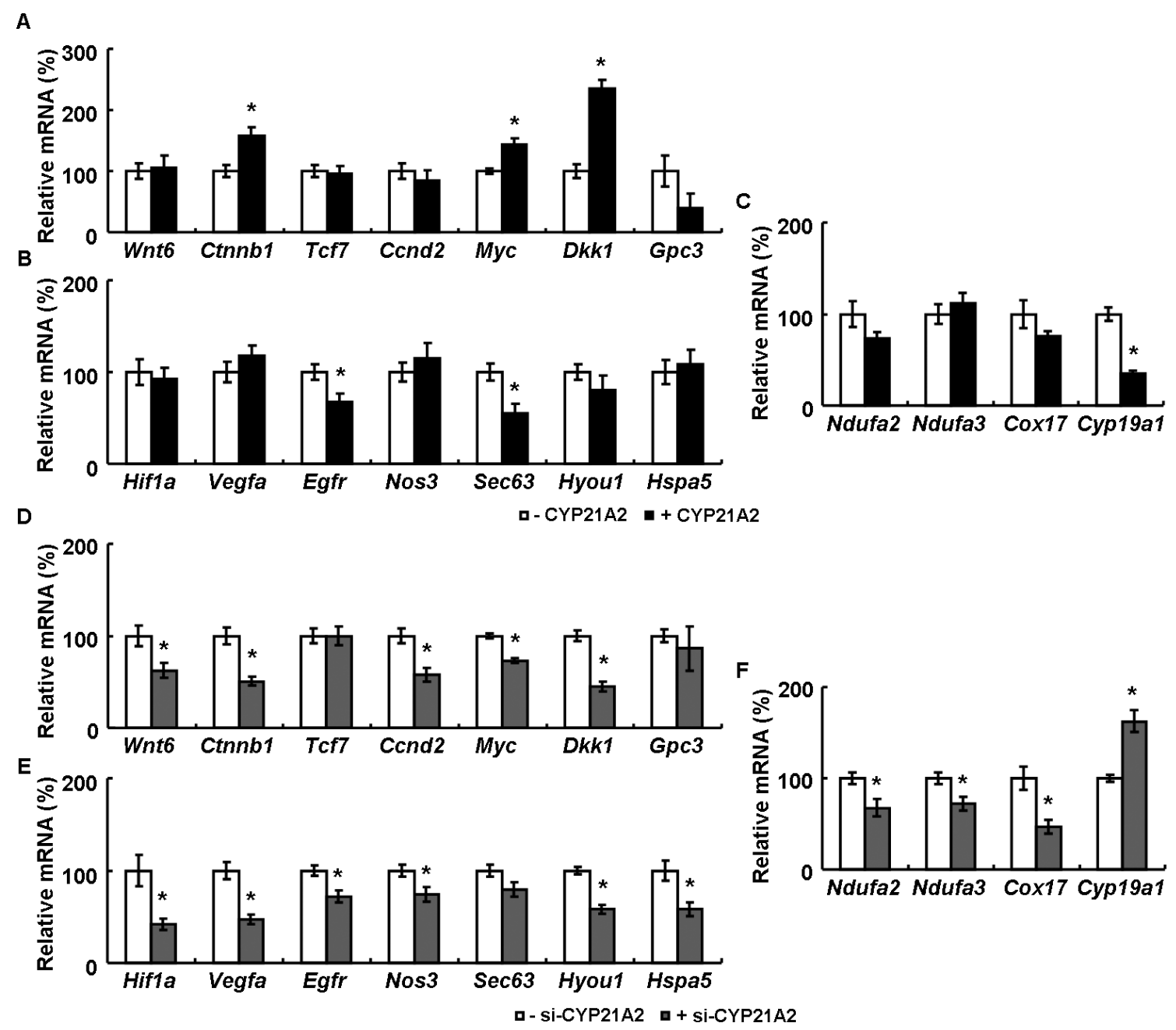

Figure 5. CYP21A2 regulates the expression of genes related to cell migration and invasion. Cells were transfected with CYP21A2 overexpressing plasmid (+ CYP21A2), control vector (- CYP21A2) for 24 h, respectively. A, D. Wnt6, Ctnnb1, Tcf7, Ccnd2, Myc, Dkk1, Gpc3 mRNA levels. B, E. Hif1a, Vegfa, Egfr, Nos3, Sec63, Hyou1, Hspa5 mRNA levels. C, F. Ndufa2, Ndufa3, Cox17, Cyp19a1 mRNA levels. Cells were transfected with CYP21A2 siRNA (+ si-CYP21A2), control siRNA (- si-CYP21A2) for 24 h, respectively. Data were obtained with at least two independent in vitro experiments ( $n=6$ per group) and presented as means \pm SEM. Statistical significance was calculated using two-tailed Student's $t$-test for the effects of CYP21A2 versus control vector, si-CYP21A2 versus control siRNA. ${ }^{*} p<0.05$. 
are poorly understood. Here, we showed that CYP21A2 overexpression or silenced did not affected cell viability or apoptosis. Furthermore, CYP21A2 silenced changed cell cycle distribution, decreased the G0/G1 phase and increased the S phase respectively, while CYP21A2 overexpressed displayed no difference in cell cycle distribution. In addition, CYP21A2 over-expression improved the abilities of cell migration and invasion, whereas CYP21A2 silenced had the opposite effects. Finally, gene expression analysis showed that CYP21A2 improved the Wnt signaling. These results highlight a critical role of CYP21A2 in the regulation of cell migration and invasion, which are important to cancer development.

Tumor and cancer development is a multistep process, influencing by lots of regulators (Kim and Roberts 2016; Luo et al. 2018; Srivastava et al. 2019). Our results exhibited that CYP21A2 mainly affected cell migration and invasion but not cell viability or apoptosis. Cell migration and invasion are complex and fundamental biological processes in life, they are critical to many pathological processes, including embryonic morphogenesis, tissue repair and regeneration, mental retardation, chronic inflammatory diseases, and cancer (Condeelis and Pollard 2006; Slattum and Rosenblatt 2014). Migration and invasion helps cancer cells invade to colonize distant metastatic lesions (Talmadge and Fidler 2010). We speculated CYP21A2 may regulated cancer formation and development via affecting cell migration and invasion. On the other hand, we found that CYP21A2 silenced changed cell cycle distribution but had no effect while overexpressed. Cell cycle distribution is important to cell proliferation, which is influenced by many stimulants and is improved dramatically in the development of cancer (Wee and Wang 2017; Hidalgo et al. 2019). Our results suggested that CYP21A2 partly affected cell cycle and may function in a complex manner.

The tumor suppressor p53 is a well known transcription factor involved in cancer (Kruiswijk et al. 2015). Previous study found that CYP21A2 was a novel target gene of p53 (Charni et al. 2016), which hinted that CYP21A2 may have a regulated role in cancer. In our study, we verified this possibility and found that some related moleculars in Wnt pathway changed, like Ctnnb1, Myc and Dkk1. Wnt signaling plays a very important role in the development of cancers (Ghosh et al. 2019). These results suggested CYP21A2 displayed its role in regulating cell migration and invasion via affecting Wnt signaling genes. This provides new evidence for the transcriptional regulation of Wnt signaling by CYP21A2.

Interestingly, we found that CYP21A2 regulated the expression of Cyp19a1, an important gene in cell oxidation which is related to oxidative phosphorylation in mitochondria (Singh et al. 2017). Previous studies demonstrated that mitochondrial respiration was essential for tumorigenesis (Weinberg et al. 2010). What's more, in the promoter of
CYP21A2, there exists a regulatory DNA element necessary for the cAMP expression and also has CREB binding site (Watanabe et al. 1993) that are important regulators to metabolism, including glucose metabolism, lipid metabolism and energy metabolism (Zhang et al. 2010). The possible involvement of CYP21A2 in metabolism requires to be studied in the future.

In summary, as described above, we observed CYP21A2 played an important role in regulating tumor- related processes and the Wnt signaling. These results provide novel insights for the potential functions and molecular mechanisms of CYP21A2 and even the treatment targets for cancer.

Acknowledgment. This work was supported by the National Natural Science Foundation (grant No. 81360138).

Conflict of interest. The authors report no conflict interest.

Author contribution statement. YG researched data, wrote, reviewed and edited the manuscript. AR, ZW, WZ and PZ researched data. FH directed the project, contributed to discussion and wrote, reviewed, and edited the manuscript. FH is the guarantor of this work and, as such, had full access to all the data in the study and takes responsibility for the integrity of the data and the accuracy of the data analysis.

\section{References}

Bernhardt R (2006): Cytochromes P450 as versatile biocatalysts. J. Biotechnol. 124, 128-145 https://doi.org/10.1016/j.jbiotec.2006.01.026

Brixius-Anderko S, Schiffer L, Hannemann F, Janocha B, Bernhardt R (2015): A CYP21A2 based whole-cell system in Escherichia coli for the biotechnological production of premedrol. Microb. Cell Fact. 14, 135

https://doi.org/10.1186/s12934-015-0333-2

Bry-Gauillard H, Cartes A, Young J (2014): Mitotane for 21-hydroxylase deficiency in an infertile man. New Engl. J. Med. 371, 2042-2044 https://doi.org/10.1056/NEJMc1410041

Calcinotto A, Kohli J, Zagato E, Pellegrini L, Demaria M, Alimonti A (2019): Cellular senescence: aging, cancer, and injury. Physiol. Rev. 99, 1047-1078 https://doi.org/10.1152/physrev.00020.2018

Canturk C, Baade U, Salazar R, Storm N, Portner R, Hoppner W (2011): Sequence analysis of CYP21A1P in a German population to aid in the molecular biological diagnosis of congenital adrenal hyperplasia. Clin. Chem. 57, 511-517 https://doi.org/10.1373/clinchem.2010.156893

Carmina E, Dewailly D, Escobar-Morreale HF, Kelestimur F, Moran C, Oberfield S, Witchel SF, Azziz R (2017): Non-classic congenital adrenal hyperplasia due to 21-hydroxylase deficiency revisited: an update with a special focus on adolescent and adult women. Hum. Reprod. Update 23, 580-599 https://doi.org/10.1093/humupd/dmx014 
Charni M, Molchadsky A, Goldstein I, Solomon H, Tal P, Goldfinger N, Yang P, Porat Z, Lozano G, Rotter V (2016): Novel p53 target genes secreted by the liver are involved in non-cell-autonomous regulation. Cell Death Differ. 23, 509-520 https://doi.org/10.1038/cdd.2015.119

Clarke R, Cook KL, Hu R, Facey CO, Tavassoly I, Schwartz JL, Baumann WT, Tyson JJ, Xuan J, Wang Y, et al. (2012): Endoplasmic reticulum stress, the unfolded protein response, autophagy, and the integrated regulation of breast cancer cell fate. Cancer Res. 72, 1321-1331 https://doi.org/10.1158/0008-5472.CAN-11-3213

Condeelis J, Pollard JW (2006): Macrophages: obligate partners for tumor cell migration, invasion, and metastasis. Cell 124, 263-266 https://doi.org/10.1016/j.cell.2006.01.007

Cooper J, Giancotti FG (2019): Integrin signaling in cancer: mechanotransduction, stemness, epithelial plasticity, and therapeutic resistance. Cancer Cell 35, 347-367 https://doi.org/10.1016/j.ccell.2019.01.007

De Palma M, Biziato D, Petrova TV (2017): Microenvironmental regulation of tumour angiogenesis. Nat. Rev. Cancer 17, 457 474 https://doi.org/10.1038/nrc.2017.51

Eifler K, Vertegaal ACO (2015): SUMOylation-mediated regulation of cell cycle progression and cancer. Trends Biochem. Sci. 40, 779-793 https://doi.org/10.1016/j.tibs.2015.09.006

El-Maouche D, Arlt W, Merke DP (2017): Congenital adrenal hyperplasia. Lancet 390, 2194-2210 https://doi.org/10.1016/S0140-6736(17)31431-9

Fulda S, Galluzzi L, Kroemer G (2010). Targeting mitochondria for cancer therapy. Nat. Rev. Drug Discov. 9, 447-464 https://doi.org/10.1038/nrd3137

Ghosh N, Hossain U, Mandal A, Sil PC (2019): The Wnt signaling pathway: a potential therapeutic target against cancer. Ann. NY Acad. Sci. 1443, 54-74 https://doi.org/10.1111/nyas.14027

Girvan HM, Munro AW (2016): Applications of microbial cytochrome P450 enzymes in biotechnology and synthetic biology. Curr. Opin. Chem. Biol. 31, 136-145 https://doi.org/10.1016/j.cbpa.2016.02.018

Green DR, Galluzzi L, Kroemer G (2014): Cell biology. Metabolic control of cell death. Science 345, 1250256 https://doi.org/10.1126/science.1250256

Hahn WC, Weinberg RA (2002): Rules for making human tumor cells. New Engl. J. Med. 347, 1593-1603 https://doi.org/10.1056/NEJMra021902

Haider S, Islam B, D‘Atri V, Sgobba M, Poojari C, Sun L, Yuen T, Zaidi M, New MI (2013): Structure-phenotype correlations of human CYP21A2 mutations in congenital adrenal hyperplasia. Proc. Nat. Acad. Sci. USA 110, 2605-2610 https://doi.org/10.1073/pnas.1221133110

Hidalgo A, Chilvers ER, Summers C, Koenderman L (2019): The neutrophil life cycle. Trends Immunol. 40, 584-597 https://doi.org/10.1016/j.it.2019.04.013

Kim KH, Roberts CW (2016): Targeting EZH2 in cancer. Nat. Med. 22, 128-134 https://doi.org/10.1038/nm.4036
Kominami S, Ochi H, Kobayashi Y, Takemori S (1980): Studies on the steroid hydroxylation system in adrenal cortex microsomes. Purification and characterization of cytochrome P-450 specific for steroid C-21 hydroxylation. J. Biol. Chem. 255, 3386-3394

Kruiswijk F, Labuschagne CF, Vousden KH (2015): p53 in survival, death and metabolic health: a lifeguard with a licence to kill. Nat. Rev. Mol. Cell Biol. 16, 393-405 https://doi.org/10.1038/nrm4007

Lamb DC, Waterman MR (2013): Unusual properties of the cytochrome P450 superfamily. Philos. Trans. R. Soc. Lond. B Biol. Sci. 368, 20120434 https://doi.org/10.1098/rstb.2012.0434

Lee KM, Giltnane JM, Balko JM, Schwarz LJ, Guerrero-Zotano AL, Hutchinson KE, Nixon MJ, Estrada MV, Sanchez V, Sanders ME, et al. (2017): MYC and MCL1 cooperatively promote chemotherapy-resistant breast cancer stem cells via regulation of mitochondrial oxidative phosphorylation. Cell Metab. 26, 633-647 e637 https://doi.org/10.1016/j.cmet.2017.09.009

Lito P, Rosen N, Solit DB (2013): Tumor adaptation and resistance to RAF inhibitors. Nature Medicine 19, 1401-1409 https://doi.org/10.1038/nm.3392

Lu X, Wang L, Lin X, Huang J, Charles Gu C, He M, Shen H, He J, Zhu J, Li H, et al. (2015): Genome-wide association study in Chinese identifies novel loci for blood pressure and hypertension. Hum. Mol. Genet. 24, 865-874

Luo C, Hajkova P, Ecker JR (2018): Dynamic DNA methylation: In the right place at the right time. Science 361, 1336-1340 https://doi.org/10.1126/science.aat6806

Martincorena I, Raine KM, Gerstung M, Dawson KJ, Haase K, Van Loo P, Davies H, Stratton MR, Campbell PJ (2018): Universal patterns of selection in cancer and somatic tissues. Cell 173, 1823 https://doi.org/10.1016/j.cell.2018.06.001

Meurette O, Mehlen P (2018): Notch signaling in the tumor microenvironment. Cancer Cell 34, 536-548 https://doi.org/10.1016/j.ccell.2018.07.009

Mizrachi D, Wang Z, Sharma KK, Gupta MK, Xu K, Dwyer CR, Auchus RJ (2011): Why human cytochrome P450c21 is a progesterone 21-hydroxylase. Biochemistry 50, 3968-3974 https://doi.org/10.1021/bi102078e

Neagu M, Constantin C, Popescu ID, Zipeto D, Tzanakakis G, Nikitovic D, Fenga C, Stratakis CA, Spandidos DA, Tsatsakis AM (2019): Inflammation and metabolism in cancer cellmitochondria key player. Front. Oncol. 9, 348 https://doi.org/10.3389/fonc.2019.00348

New MI, Abraham M, Gonzalez B, Dumic M, Razzaghy-Azar M, Chitayat D, Sun L, Zaidi M, Wilson RC, Yuen T (2013): Genotype-phenotype correlation in 1,507 families with congenital adrenal hyperplasia owing to 21-hydroxylase deficiency. Proc. Nat. Acad. Sci. USA 110, 2611-2616 https://doi.org/10.1073/pnas.1300057110

Puisieux A, Pommier RM, Morel AP, Lavial F (2018): Cellular pliancy and the multistep process of tumorigenesis. Cancer Cell 33, 164-172 https://doi.org/10.1016/j.ccell.2018.01.007

Rojo de la Vega M, Chapman E, Zhang DD (2018): NRF2 and the hallmarks of cancer. Cancer Cell 34, 21-43 https://doi.org/10.1016/j.ccell.2018.03.022 
Ryan KJ, Engel LL (1957): Hydroxylation of steroids at carbon 21. J. Biol. Chem. 225, 103-114

Schmitt AM, Chang HY (2016): Long noncoding RNAs in cancer pathways. Cancer Cell 29, 452-463 https://doi.org/10.1016/j.ccell.2016.03.010

Siegel RL, Miller KD, Jemal A (2019): Cancer statistics, 2019. CA Cancer J. Clin. 69, 7-34 https://doi.org/10.3322/caac.21551

Singh V, Ram M, Kumar R, Prasad R, Roy BK, Singh KK (2017): Phosphorylation: implications in cancer. The Protein Journal 36, 1-6 https://doi.org/10.1007/s10930-017-9696-Z

Slattum GM, Rosenblatt J (2014): Tumour cell invasion: an emerging role for basal epithelial cell extrusion. Nat. Rev. Cancer 14, 495-501 https://doi.org/10.1038/nrc3767

Srivastava S, Koay EJ, Borowsky AD, De Marzo AM, Ghosh S, Wagner PD, Kramer BS (2019): Cancer overdiagnosis: a biological challenge and clinical dilemma. Nat. Rev. Cancer 19, 349-358 https://doi.org/10.1038/s41568-019-0142-8

Strilic B, Offermanns S (2017): Intravascular survival and extravasation of tumor cells. Cancer Cell 32, 282-293 https://doi.org/10.1016/j.ccell.2017.07.001

Talmadge JE, Fidler IJ (2010): AACR centennial series: the biology of cancer metastasis: historical perspective. Cancer Res. 70, 5649-5669 https://doi.org/10.1158/0008-5472.CAN-10-1040

VanderVorst K, Dreyer CA, Konopelski SE, Lee H, Ho HH, Carraway KL, 3rd (2019): Wnt/PCP signaling contribution to carcinoma collective cell migration and metastasis. Cancer Res. 79, 1719-1729
https://doi.org/10.1158/0008-5472.CAN-18-2757

Vogelstein B, Lane D, Levine AJ (2000): Surfing the p53 network. Nature 408, 307-310

https://doi.org/10.1038/35042675

Watanabe N, Kitazume M, Fujisawa J, Yoshida M, Fujii-Kuriyama Y (1993). A novel cAMP-dependent regulatory region including a sequence like the cAMP-responsive element, far upstream of the human CYP21A2 gene. Eur. J. Biochem. 214, 521-531 https://doi.org/10.1111/j.1432-1033.1993.tb17950.x

Wee P, Wang Z (2017): Cell cycle synchronization of HeLa cells to assay EGFR pathway activation. Methods Mol. Biol. 1652, $167-181$ https://doi.org/10.1007/978-1-4939-7219-7_13

Weinberg F, Hamanaka R, Wheaton WW, Weinberg S, Joseph J, Lopez M, Kalyanaraman B, Mutlu GM, Budinger GR, Chandel NS (2010): Mitochondrial metabolism and ROS generation are essential for Kras-mediated tumorigenicity. Proc. Nat. Acad. Sci. USA 107, 8788-8793 https://doi.org/10.1073/pnas.1003428107

Zhang EE, Liu Y, Dentin R, Pongsawakul PY, Liu AC, Hirota T, Nusinow DA, Sun X, Landais S, Kodama Y, et al. (2010): Cryptochrome mediates circadian regulation of cAMP signaling and hepatic gluconeogenesis. Nat. Med. 16, 1152-1156 https://doi.org/10.1038/nm.2214

Zhu J, Thompson CB (2019): Metabolic regulation of cell growth and proliferation. Nat. Rev. Mol. Cell Biol. 20, 436-450 https://doi.org/10.1038/s41580-019-0123-5

Received: August 23, 2019

Final version accepted: January 21, 2020 
Supplementary Material

\title{
A novel function of CYP21A2 in regulating cell migration and invasion via Wnt signaling
}

\author{
Yajie Guo ${ }^{1}$, Aliya Rehati ${ }^{2}$, Zhijing $\mathrm{Wu}^{1}$, Wenyuan Zhang $^{1}$, Peng Zhuang ${ }^{1}$ and Fangping $\mathrm{He}^{1}$ \\ 1 The Eighth Affiliated Hospital, Sun Yat-Sen University, Shenzhen, Guangdong province, China \\ 2 The First Affiliated Hospital of Xinjiang Medical University, Department of Hepatology, Urumchi, Xinjiang province, China
}

Supplementary Table

Table S1. List of oligonucleotide primer pairs used in RT-PCR analysis

\begin{tabular}{|l|l|l|}
\hline Gene & Forward primer (5'-3') & Reverse primer (5'-3') \\
\hline Hsa-Actin & CATGTACGTTGCTATCCAGGC & 'TCCTTAATGTCACGCACGAT \\
\hline Has-Cyp21a2 & TCATACTGGGGTGCGGAGA & AGATGGGACATCCGGCTTTG \\
\hline Hsa-Wnt6 & GGGAGCGTTAAAGGACACT & ACTAACCTCACCCACCATCCT \\
\hline Hsa-Ctnnb1 & AGCAGGTGGATCTATTTCATGT & GCAGGTTACAACAACTTTGGGA \\
\hline Hsa-Tcf7 & GCGGGACAGAGGACCATTAC & TCAAGGCTGAGGGTTGAAGC \\
\hline Hsa-Ccnd2 & TGCAGCCTACTTTGGGGAA & CCAATGGCCCGATGATTTGC \\
\hline Hsa-Myc & TGTCCTGAGCAATCACCTATGA & CCCAAAGTCCAATTTGAGCA \\
\hline Hsa-Dkk1 & GTGGTTTCAGTTAAGCATTCCA & ACAGGTAAGTGCCACACTGAG \\
\hline Hsa-Gpc3 & GCATTGGAGGCTCTGGTGAT & GTTGCCTGCTGACTGTTTCC \\
\hline Hsa-Hif1a & TCCTTTTGCTCTTTGTGGTTGGA & GCCTGGTCCACAGAAGATGTTTA \\
\hline Hsa-Vegfa & TGTGGAGGCAGAGAAAAGAGA & AGAGAGCAAGAGAGAGCAAAAGA \\
\hline Hsa-Egfr & GCTAGTTAGGAGCCCACCTTTT & CTTGATAAATTGGATGGGGTGG \\
\hline Hsa-Nos3 & ACCCGCTTCCTGTTTCTTAGT & AATCCACATGAGCTGGGGTAG \\
\hline Hsa-Sec63 & TTCCCACAAACGGTCTGATTT & TGCTTGGTGCTAACTTCTTGAG \\
\hline Hsa-Hyou1 & GTGTCTGCAGAGCCTCAGTT & GAAACCAGGGGGAAGGAG \\
\hline Hsa-Hspa5 & GTGTACCCTGGGGCAATAGG & CAGTAACAGCTCCTCAGAAAGA \\
\hline Hsa-Ndufa2 & TGGCCAAGAGACGAATGTCC & ACTAAGTCCAGCAGAGCCCA \\
\hline Hsa-Ndufa3 & ACCTGGAGTCCCTGAATAAAGA & GGGATAAACCAGACAAGTAGGCT \\
\hline Hsa-Cox17 & TCAGGAGAAGAAGCCGCTGAAG & ATCCTAGGGCTCTCATGCATTC \\
\hline Has-Cyp19a1 & TGGCATGTTCCCATTTTTGCTT & GTTTGAGCCCATTGGGTTGG \\
\hline
\end{tabular}

Supplementary Figures

A

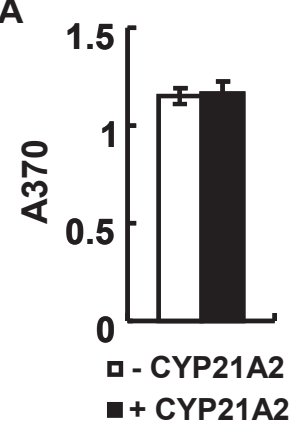

B

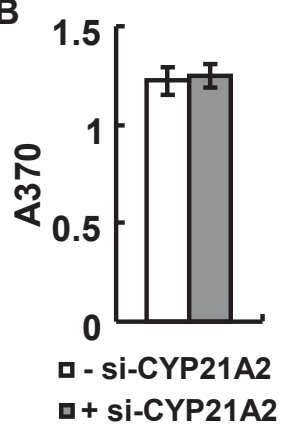

Figure S1. Effects of CYP21A2 on proliferation of cells. A. Results of absorbance at $370 \mathrm{~nm}$. Cells were transfected with CYP21A2 overexpressing plasmid (+ CYP21A2), control vector (-CYP21A2) for $24 \mathrm{~h}$, respectively. B. Results of absorbance at $370 \mathrm{~nm}$. Cells were transfected with CYP21A2 siRNA (+ si-CYP21A2), control siRNA (-si-CYP21A2) for $24 \mathrm{~h}$, respectively. Data were obtained with at least three independent in vitro experiments ( $n=6$ per group) and presented as means \pm SEM. Statistical significance was calculated using two-tailed Student $t$-test for the effects of CYP21A2 versus control vector, si-CYP21A2 versus control siRNA. 
A
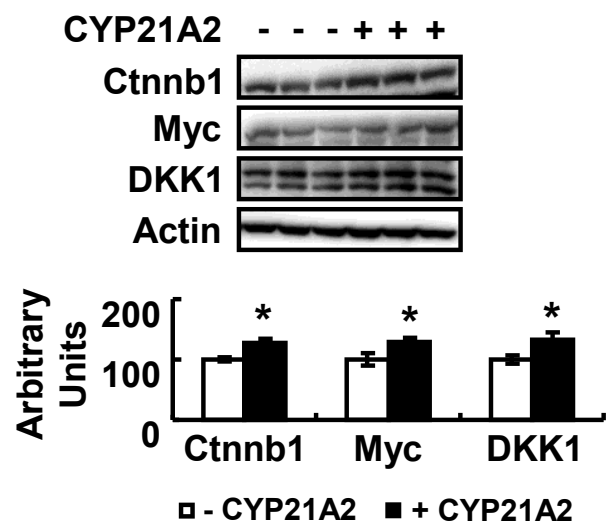

B
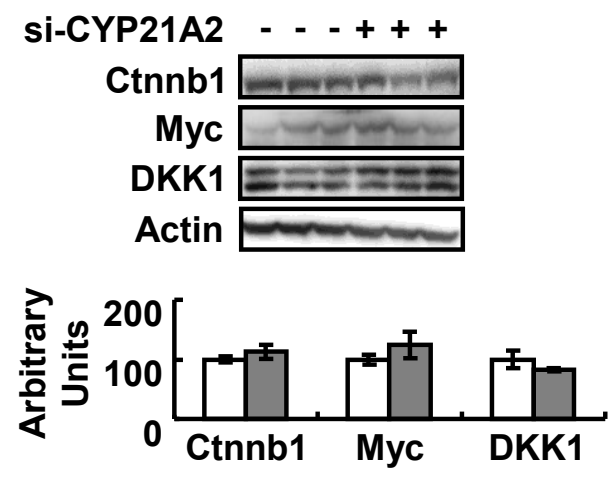

a-si-CYP21A2 a + si-CYP21A2

Figure S2. Effects of CYP21A2 on Wnt signaling genes expression. A. Ctnnb1, Myc and DKK1 protein (top, western blot; bottom, quantitative measurements of Ctnnb1, Myc and DKK1 protein relative to actin). Cells were transfected with CYP21A2 overexpressing plasmid (+ CYP21A2), control vector (- CYP21A2) for $24 \mathrm{~h}$, respectively. B. Ctnnb1, Myc and DKK1 protein (top, Western blot; bottom, quantitative measurements of Ctnnb1, Myc and DKK1 protein relative to actin). Cells were transfected with CYP21A2 siRNA (+ siCYP21A2), control siRNA (- si-CYP21A2) for $24 \mathrm{~h}$, respectively. Data were obtained with at least two independent in vitro experiments ( $n=6$ per group) and presented as means \pm SEMs. Statistical significance was calculated using two-tailed student $t$-test for the effects of CYP21A2 versus control vector, si-CYP21A2 versus control siRNA $\left({ }^{*} p<0.05\right)$. 